\title{
Models, postulates, and generalized nomic truth approximation
}

\author{
Theo A. F. Kuipers ${ }^{1}$
}

Received: 10 February 2015 / Accepted: 14 September 2015 / Published online: 28 September 2015 (C) The Author(s) 2015. This article is published with open access at Springerlink.com

\begin{abstract}
The qualitative theory of nomic truth approximation, presented in Kuipers in his (from instrumentalism to constructive realism, 2000), in which 'the truth' concerns the distinction between nomic, e.g. physical, possibilities and impossibilities, rests on a very restrictive assumption, viz. that theories always claim to characterize the boundary between nomic possibilities and impossibilities. Fully recognizing two different functions of theories, viz. excluding and representing, this paper drops this assumption by conceiving theories in development as tuples of postulates and models, where the postulates claim to exclude nomic impossibilities and the (not-excluded) models claim to represent nomic possibilities. Revising theories becomes then a matter of adding or revising models and/or postulates in the light of increasing evidence, captured by a special kind of theories, viz. 'data-theories'. Under the assumption that the data-theory is true, achieving empirical progress in this way provides good reasons for the abductive conclusion that truth approximation has been achieved as well. Here, the notions of truth approximation and empirical progress are formally direct generalizations of the earlier ones. However, truth approximation is now explicitly defined in terms of increasing truth-content and decreasing falsity-content of theories, whereas empirical progress is defined in terms of lasting increased accepted and decreased rejected content in the light of increasing evidence. These definitions are strongly inspired by a paper of Gustavo Cevolani, Vincenzo Crupi and Roberto Festa, viz., "Verisimilitude and belief change for conjunctive theories" (Cevolani et al. in Erkenntnis 75(2):183-222, 2011).
\end{abstract}

\footnotetext{
Theo A. F. Kuipers

t.a.f.kuipers@rug.nl

1 Philosophy of science, University of Groningen, Groningen, The Netherlands
} 
Keywords Models · Postulates · Nomic truth approximation · Empirical progress · Theory revision $\cdot$ Two-sided theories

\section{Introduction}

The idea of truthlikeness or verisimilitude amounts to the claim that one theory may be closer, or more similar, to the truth than another. It was made an interesting topic in the philosophy of science by Popper (1963), who presented a very plausible, but failing explication of it. He proposed that 'closer to the truth' holds when the one theory has more true and less false consequences than the other. Miller (1974) and Tichý (1974) independently proved that a false theory, in the sense of a theory with at least one false consequence, could according to Popper's definition never be closer to the truth than another one. Ever since, there have been developed other accounts that circumvent this problem successfully. See Kuipers (1987) for an incomplete collection of approaches. See Niiniluoto (1998) for an important survey and Oddie (2014) for the most recent survey. Some global distinctions are in order. (1) Authors may put the emphasis on a quantitative definition, based on a distance-from-the-truth measure, notably Niiniluoto (1987), or on a qualitative definition of the comparative closer-tothe-truth claim (e.g. Oddie 1986; Zwart 2001). It is important to note that in a qualitative approach most theories will be incomparable, that is, in most cases one theory will be neither closer, nor less close, to the truth than another. The advantage of such an approach is that it focusses on safe cases of comparison, providing a sound point of departure for concretizations, e.g. a quantitative approach. (2) As a rule, authors focus on (truthlikeness with respect to) 'the actual truth', that is, the truth about what is (or was) actually the case. However, from a philosophy of science point of view, one may argue that scientists are aiming at theories that capture what is physically possible and what is not. In other words, they are aiming at 'the physical truth' or, more broadly, 'the nomic truth', which makes nomic truthlikeness the topic of investigation. (3) Finally, usually authors opt for a logical approach and conceive theories primarily as sets of sentences or propositions, but one may also opt for a structuralist approach and conceive theories primarily as sets of conceptual possibilities, represented by models or, more generally, by set-theoretic structures.

The 'nomic truth' can perhaps best be illustrated by my favorite toy example of theory oriented science (see Kuipers 2000, p. 143). To represent an electric circuit with several switches and bulbs one may use a language with elementary propositions that enable to indicate which switches are on and which are off and also to indicate which bulbs give light and which do not. Several of the conceptually possible states will be physically possible, and one of them will be the actual state.

Referring to Fig. 1, let $p_{i}$ for $1 \leq i \leq 4$ indicate that switch $i$ is on $(\diamond)$ and $\neg p_{i}$ that it is off $(\bowtie)$. Let $q(\neg q)$ indicate that the bulb lights (does not light). It is assumed that the bulb is not defective and that there is enough voltage. A possible state of the circuit can be represented by a conjunction of negated and un-negated $p_{i}$ 's. It is clear that there is just one true description of the actual state of the circuit as it is depicted, $q \& p_{1} \& \neg p_{2} \& p_{3} \& p_{4}$, according to the standard propositional representation. Hence, the example nicely illustrates, among others, that we consider 'the actual world' pri- 
Fig. 1 An electric circuit

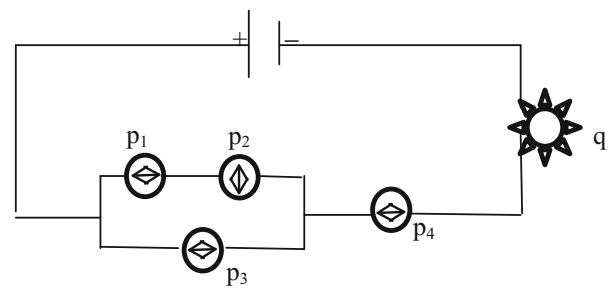

marily as something partial and local, i.e., one or more aspects of a small part of the actual universe. However, it need not be restricted to a momentary state, it may also concern an actual trajectory of states in a certain time interval. In sum, the actual world is the actual world in a certain context. To represent the physically possible states by one proposition or theory one will have to design a complex proposition, the nomic truth, in the above case:

$$
q \leftrightarrow[(p 1 \& p 2) \text { or } p 3] \& p 4
$$

All states in which this proposition is true are physically possible, all others are not. All propositions that can be formulated in the indicated language may be considered as candidates for being this nomic truth and, at least intuitively, one proposition may be closer to the truth than another, viz. when it captures more physically possible states and less impossible ones. This may be a toy example for representing theory oriented science, but in present day epigenetics there is a close analogy: in fact, genes are considered as switches that may be on or off. However this may be, only the general tenet of the example is relevant: theory oriented science is ultimately aiming at characterizing what is e.g. physically, biologically or economically possible, and theories are tested by experiments which are realizations of possibilities.

In Kuipers (2000) I have presented a qualitative theory of nomic truth approximation in a structuralist way, in which 'the nomic truth' is more specifically conceived of as the true boundary between nomic, e.g. physical, biological, etc., possibilities and impossibilities within a target domain and a theory amounts to a classification of all conceptual possibilities as either nomically possible or nomically impossible. In the basic form of my account, a theory is defined as closer to the truth than another when it makes less classification mistakes, not merely in numbers, but in the strong sense that all its classification mistakes are shared by the other theory, which makes in addition some extra mistakes.

However, it recently turned out that the motivation of this formal definition is based on a very restrictive, but unnecessary assumption, namely that theories give a complete classification of all conceptual possibilities. In Kuipers (2014b) I have shown that the formal definition can already be motivated when a theory is only supposed to claim that it includes all nomic possibilities, and hence that all its non-members are nomic impossibilities. This new motivation is in terms of increasing truth-content and decreasing falsity-content and is strongly stimulated by a paper of Cevolani et al. (2011). Formally, it is not difficult to see that the formal definition can also be motivated in a similar way by assuming that a theory only claims that all its members are nomic possibilities (instead of claiming that it includes all nomic possibilities). 
This paper exploits the possibility of dropping the restrictive assumption in a conceptually very attractive way by combining both indicated ways. The resulting theory of nomic truth approximation becomes conceptually in complete harmony with two prima facie opposing functions of theories. On the one hand, there is the Popperian or exclusion view that theories exclude certain (conceptual) possibilities from occurring or being realizable. On the other hand, there is the inclusion or representation view that theories represent, in relevant respects, certain possibilities as realized or realizable. ${ }^{1}$ The exclusion function is typically associated with speaking of the axioms, principles or postulates of theories. They have to be satisfied and hence they exclude together everything which does not satisfy them all. The representation function on the other hand is typically associated with speaking of (specified) models of theories. Seen from this perspective, I assume in Kuipers (2000) that the set of (representing) models of a theory coincides with the set of models, in the formal sense, of the (excluding) postulates of the theory. Although such a 'maximal' theory may be the ultimate aim of nomic theorizing, viz. the strongest true theory, it is not realistic to assume this of 'theories in development'. In sum, dropping the restrictive assumption makes it perfectly possible to separate models and postulates, in order to fully recognize the twofold function of theories in general and in aiming at truth approximation in particular.

Hence, in this paper a theory will in general be taken as 'non-maximal' in the following two-sided sense, viz. as a combination of a set of Models and a set of Postulates, where the former have to satisfy the latter, but need not exhaust the set of models of the joint Postulates. It will be convenient to represent the Postulates by the set of all its models: models (Postulates). Hence, a theory becomes a tuple of the following form:

$<\mathrm{M}, \mathrm{P}>$ with $\mathrm{P}=$ models(Postulates) and $\mathrm{M}$ (odels) being a subset of $\mathrm{P}$

This two-sided approach to theories can do justice to three different views in philosophy of science, viz. theorizing is mainly a matter of (1) formulating and revising postulates, or (2) designing and redesigning models, or (3) the two-sided combination of them. The generalized theory of truth approximation to follow will provide additional support for the two-sided view, but will leave room for both one-sided views.

It is important to note, as an aside, that the two-sided view on theories is also in perfect agreement with the hypothetical-deductive (HD) and deductive-nomological (DN) views on prediction and explanation, respectively. For the prediction and explanation of an event we start with representing (modelling) the situation in the relevant terms, as far as possible, but without the crucial event. This is derived by applying the relevant postulates, which amounts to completing or closing the model as far as required. Hence, prediction and explanation naturally appear as co-production of (partial) models and postulates, that is, of representation and exclusion.

The generalized theory of truth approximation presented below is based on the twosided view on theories and technically reduces to that of Kuipers (2000) by assuming $\mathrm{M}=\mathrm{P}$ throughout. The representation claim will be associated with $\mathrm{M}$ and the exclusion claim with P. Truth approximation is construed in terms of increasing truth-content

1 The representation by theories in development will usually be in an approximate way, due to idealizations. 
and decreasing falsity-content of the claims by adding or revising models and/or postulates in the light of increasing evidence. The generalized theory further follows Kuipers (2000) in reconstructing evidence as a 'data-theory' based on realized, hence nomic, possibilities and inductive generalizations based on them (and implying induced impossibilities). The evidence will guide the comparative assessment of the success of theories and the subsequent planning of new experiments to be performed leading to increasing evidence. Ultimately, the comparative success assessment may give good reasons not only for the inductive conclusion that empirical progress has been made by a revised version of the theory relative to the original one but even for the abductive conclusion that it is closer to the truth than the original and hence that truth approximation has been achieved.

We start with presenting an example of a two-sided theory (Sect. 2) and then outline the essentials of any theory of (nomic) truth approximation (Sect. 3). A relatively detailed presentation of the generalized theory follows (Sects. 4-7), with emphasis on what is conceptually new relative to Kuipers (2000). We close by indicating a number of perspectives on concretization, an alternative interpretation, and a link with belief revision (Sect. 8).

\section{An example of a two-sided theory}

The following, simplified, example illustrates the idea of a two-sided theory. Newton's theory can be represented on the one hand by the three laws of motion, i.e. by its (general) postulates, and on the other by various sets of models, e.g. (cpm-)models of classical particles mechanics (cpm) and models of classical rigid body mechanics, with specific sub-classes.

Focusing on the set or universe of conceptually possible systems of classical particle mechanics, $\mathrm{U}_{\mathrm{cpm}}$, the general postulates determine together the set of all models satisfying them, $\mathrm{P}_{\mathrm{cpm}}\left(\subseteq \mathrm{U}_{\mathrm{cpm}}\right)$. Various sub-classes of $\mathrm{P}_{\mathrm{cpm}}$ are based on specific assumptions about the nature of such systems, notably so-called special force laws, e.g. the set of models $\mathrm{P}_{\text {gcpm }}$ satisfying the law of gravitation, but also on many system specific assumptions. Hence, a specific model for a specific system is built up by starting bottom-up with system specific details and is top-down completed by applying the general and the special postulates, here called the GSP-closure of the model. Such a specific model may or may not be generalized to a subset of models for a specific subtype of cpm-systems. ${ }^{2}$ For example, let $\mathbf{M}_{\text {fo }}$ indicate the GSP-closure of objects of medium size and weight, falling from a medium distance on the earth. ${ }^{3}$

As soon as a particular model is claimed to represent a particular system (e.g a falling brick) it predicts behavior of the system in accordance with that model. ${ }^{4}$ If that

\footnotetext{
2 Note that such a set is comparable to a set of exemplars in Kuhn's sense or a set (of GSP-closures of tentative representations) of the intended applications in Sneed's sense.

3 Subsets of $\mathrm{P}_{\mathrm{cpm}}$ corresponding to systems with different kinds of forces may overlap, notably in the case of two kinds of forces, but they need not exhaust $\mathrm{P}_{\mathrm{cpm}}$, e.g. there may be room for as yet unknown (formally different) kinds of forces.

4 This also provides a potential explanation of that behavior, where, in agreement with the DN-model of explanation, the bottom-up data function as initial conditions and the postulates as laws.
} 
Fig. 2 The classical theory of gravitational particle mechanics (still) restricted to falling objects

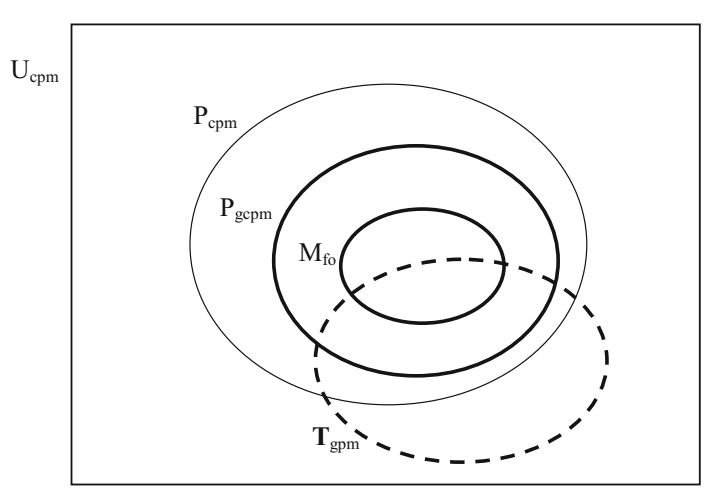

model is disconfirmed some specific (bottom-up) assumption may have to be revised, but if similar models for similar systems are also disconfirmed, the whole relevant class of particular models may have to be revised, e.g. by revising the particular force law (top-down). In this way we see that revising a two-sided theory may be a matter of adding or revising postulates that exclude things (top-down) or of adding or revising models that are supposed to represent and hence to be included (bottom-up), or both.

Let us now assume that there is a sharp, but not yet specified, distinction or boundary between the set $\mathbf{T}_{\mathrm{gpm}}$ of physically possible systems of gravitational particle mechanics, as represented in $U_{\mathrm{cpm}}$, and the set of physically impossible systems of gravitational particle mechanics, that is, the complement of $\mathbf{T}_{\mathrm{gpm}}$. Figure 2 illustrates the four subsets of $\mathrm{U}_{\mathrm{cpm}}$ introduced: $\mathrm{P}_{\mathrm{cpm}}$, the subset $\mathrm{P}_{\mathrm{gcpm}}$, and the latter's subset $\mathrm{M}_{\mathrm{fo}}$, and finally the, unknown, target set $\mathbf{T}_{\mathrm{gpm}}$.

Now we may reconstruct truth approximation as a matter of aiming at establishing the true boundary between nomic possibilities and impossibilities, or the truth about such a boundary, in the present case, starting from the two-sided theory $<\mathrm{M}_{\mathrm{fo}}, \mathrm{P}_{\mathrm{gcpm}}>$ with the claim $\mathrm{M}_{\text {fo }} \subseteq \mathbf{T}_{\text {gpm }} \subseteq \mathrm{P}_{\text {gcpm }}$, and revising it by adding or revising the models and/or adding or revising the postulates in the light of increasing evidence, with the ideal end the 'maximal and true' or the strongest true theory $<\mathrm{M}^{\#}, \mathrm{P}^{\#}>$, i.e. the theory for which $\mathrm{M}^{\#}=\mathbf{T}_{\mathrm{gpm}}=\mathrm{P}^{\#}$.

\section{Outline of a theory of (nomic) truth approximation}

The paper is set up by following the cornerstones of any theory of truth approximation as developed in Kuipers (2000) and enriched by Cevolani et al. (2011) and Cevolani et al. (2013).

I Initial step: description of the target

- Assuming a domain of research and a vocabulary, clarify the target you are aiming at, that is, about which you aim to approach the truth.

- Here, the target is the nomic truth, i.e. which conceptual possibilities are nomically possible in the domain of research and which are not. 
II Logico-semantic steps: define closer to the truth (greater verisimilitude or truthlikeness)

- Define your notion of theory, which we did already, and define next the notion of true (false) theories and hence of the strongest true theory, i.e. the truth.

- Define the notions of the truth-content and the falsity-content of a theory.

- Define closer to the truth in terms of suitably specified notions of a larger truth-content and a smaller, or otherwise less problematic, falsity-content.

III Epistemological steps: define more successful

- Identify the kind of evidence that experiments provide, up to and including inductive generalizations.

- Assuming that the evidence is accepted, define the therewith accepted content (or the successes) and rejected content (or the failures) of a theory.

- Define more successful in terms of a larger accepted content and a smaller, or otherwise less problematic, rejected content.

IV Theoretical step: from verisimilitude to success

- Assuming the truth of the (accepted) evidence, prove the strongest 'success theorem'. Ideally this theorem amounts to: 'closer to the truth' unconditionally entails 'at least as successful' and in the long run even 'more successful'.

V Methodological steps: from success to verisimilitude

- Assuming that a new theory is at a certain moment more successful than the old one, propose and test the empirical progress hypothesis: the new theory (is and) remains more successful than the old one.

- Assuming that after 'sufficient confirmation'5 the empirical progress hypothesis is accepted (for the time being), argue on the basis of the success theorem that the best explanation for this case of empirical progress is the truth approximation hypothesis that the new theory is closer to the truth than the old one, i.e. that this is a case of truth approximation.

- Abductively conclude (for the time being) that the new theory is closer to the truth than the old one, i.e. that truth approximation has been achieved.

In this paper I will put the formal emphasis on steps II and III, corresponding to Sects. 5 and 6, because they are conceptually new, whereas essentially similar versions of the remaining steps have been elaborated in Kuipers (2000). Sections 4 and 6.1 introduce the crucial points of departure from Kuipers (2000) regarding the target of nomic research and of how evidence is conceived, respectively.

\section{I Initial step: description of the target}

Let $\mathrm{U}$ indicate the set of conceptual possibilities in a given context (e.g. the possible states, trajectories or transformations ${ }^{6}$ of a system, or possible kinds of systems),

\footnotetext{
5 What sufficient confirmation is, is of course a matter of taste and may hence be a matter of debate.

6 Deutsch (2013) is drawing attention, in terms of 'constructor theory', to the importance in physics and elsewhere of the distinction between possible and impossible 'transformations'. In these terms, the present paper is dealing with two-sided truth approximation regarding the true boundary between nomically possible and nomically impossible (but conceptually possible) transformations.
} 
Fig. 3 The set of conceptual possibilities $U$ and the (unknown) subset of nomic possibilities $\mathbf{T}$

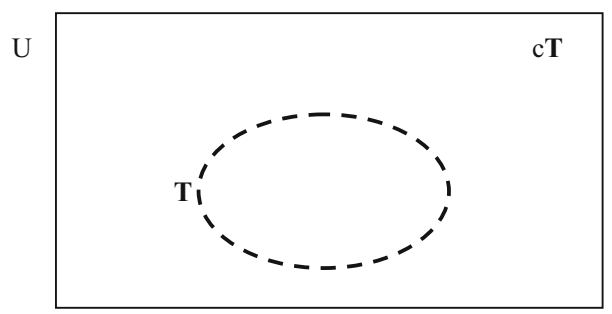

generated by a descriptive vocabulary V in which U is characterized as V's set of settheoretic structures, and in which subsets of U, e.g. X, Y, R, S, can be characterized. Complements of sets will be indicated by a preceding 'c': e.g. cX. By the way, U should not to be taken as a set of possible worlds in the standard 'there is only one, all-inclusive, world' sense. Our possibilities are relative to a certain context or (type of) system(s), sometimes called 'small worlds'. They are only mutually exclusive and jointly exhaustive in the same case in the given context, e.g. in one state of a system. In this respect they can best be compared with the possible 'elementary outcomes' of an experiment in probability theory, e.g. throwing a die: although the six faces are mutually exclusive (and jointly exhaustive) in one experiment, they are not when different experiments are considered. However, there is also an important difference: instead of the six faces, not all our conceptual possibilities are supposed to be physically possible.

Let (bold) $\mathbf{T}$ indicate the subset of nomic, e.g. physical, possibilities, and hence cT the subset of nomic impossibilities. By the bold ' $\mathbf{T}$ ' we indicate that we do not (yet) dispose of a characterization of it in terms of $\mathrm{V}$, see the dashed ellipse in Fig. 3. The target of research is identifying, if possible, T's boundary in V-terms, indicated by (non-bold) $\mathrm{T}$, hence $\mathrm{T}=\mathbf{T}$, assuming such a characterization exists, which I will do throughout in this paper. $\mathrm{T}$ will be called 'the (explicit) (nomic) truth', for reasons that will become clear.

\section{II Logico-semantic steps: define closer to the truth}

\subsection{Theories, their claims, and 'the truth'}

For the logico-semantic steps we start with defining the notion of theories. In the present nomic context, theories are intended to (at least partially) characterize $\mathbf{T}$. Recall that for that purpose a theory is conceived as a tuple $<\mathrm{M}, \mathrm{P}>$ of subsets of $\mathrm{U}$, defined in V-terms, with $\mathrm{P}=$ Models (Postulates) and the claims:

" $\mathrm{M} \subseteq \mathbf{T}$ ", the inclusion or representation claim: all members of $\mathrm{M}$ are nomic possibilities,

“T $\subseteq$ P", i.e. "cP $\subseteq$ cT", the exclusion claim: all non-members of $\mathrm{P}$ are excluded from being nomic possibilities (are nomic impossibilities), or, equivalently, no nomic possibility is excluded by the postulates of the theory. 
The members of $\mathrm{M}$ are called the models of the theory and those of $\mathrm{P}$ the models of the postulates of the theory. ${ }^{7}$

A theory is consistent if $\mathrm{M} \subseteq \mathrm{P}$, i.e. when its two claims are compatible; it is inconsistent otherwise. Note that this notion of inconsistency is not the standard logical one, but here plausible and of course related. ${ }^{8}$ This paper is restricted to consistent theories, leaving inconsistent ones for a future paper, for they seem interesting as well. A theory is maximal if $\mathrm{M}=\mathrm{P}$; it is non-maximal otherwise. Kuipers (2000) is restricted to maximal theories. Maximal theories in this sense seem also characteristic for the model-theoretic and structuralist ${ }^{9}$ or semantic view on theories.

It will be useful to also define two other extreme kinds of theories, besides maximal ones, so-called pure (or one-sided) theories. A theory $<\mathrm{M}, \mathrm{P}>$ is a pure theory of postulates, or a pure exclusion theory, if $\mathrm{M}=\varnothing$ and it is a pure theory of models, or a pure inclusion theory, if $\mathrm{P}=\mathrm{U}$. In these terms, a (non-pure or two-sided) theory $<\mathrm{M}, \mathrm{P}>$ is a combination of a pure theory of models $<\mathrm{M}, \mathrm{U}>$ and a pure theory of postulates $<\varnothing, \mathrm{P}>$, also called the M-side (or inclusion) theory and the P-side (or exclusion) theory, respectively.

Finally, a theory $<\mathrm{M}, \mathrm{P}>$ is true if both claims are true, i.e. $\mathrm{M} \subseteq \mathbf{T} \subseteq \mathrm{P}$, false otherwise. Now it is easy to see that there is at most one maximal or strongest true theory, called the true (nomic) theory or simply the (nomic) truth, viz. the one for which $\mathrm{M}=\mathbf{T}=\mathrm{P}$. From now on, 'the truth' will refer to 'the nomic truth', except when otherwise stated. It results from the characterization of $\mathbf{T}$ in $\mathrm{V}$-terms, if it exists. It will be indicated by $<\mathrm{T}, \mathrm{T}>$, or simply $\mathrm{T}$, with non-bold ' $\mathrm{T}$ '. This $\mathrm{T}$ is the target of (theory-oriented) research.

It is also easy to check that there is at most one strongest true pure theory of models, viz. $<\mathrm{T}, \mathrm{U}>$ and that there is at most one strongest true pure theory of postulates, viz. $<\varnothing, \mathrm{T}>$. Hence, $<\mathrm{T}, \mathrm{T}>$ is the strongest true (two-sided) theory.

\subsection{Truth- and falsity-content}

In view of the two claims of a theory it is plausible how to define the truth-content and the falsity-content of a theory. Consider the M-side claim $\mathbf{M} \subseteq \mathbf{T}$. As far as $\mathrm{M} \cap \mathbf{T}$

\footnotetext{
7 Although we suggest that $\mathrm{P}$ is determined as the set of models of a given set of postulates, and $\mathrm{M}$ is just given as a set of members of $\mathrm{U}$, it may well be that in some contexts both $\mathrm{P}$ and $\mathrm{M}$ are directly specified as subsets of U. Alternatively, whereas P may be based on a set of exclusion postulates, M may be based on a set of inclusion postulates. However, I prefer to speak of models because the term 'model' suggests to represent something and hence to be included in some target set, whereas the term 'postulate' suggests to exclude things by imposing a necessary condition to be satisfied in order to be included.

8 A theory is inconsistent in the syntactic (logical) sense when it is possible to derive a contradiction or in the semantic (logical), but equivalent, sense when there is no model satisfying it.

9 In the first view, the postulates (or axioms) are explicitly formulated in a specified language and the models are those structures of the language that satisfy all of them. In the second view, the language is not specified but the models are directly specified in such a way that the postulates are built into them. Note that in the basic format of a theory in the structuralist sense $<\mathrm{M}_{\mathrm{p}}, \mathrm{M}, \mathrm{I}>, \mathrm{M}_{\mathrm{p}}$ corresponds with our $\mathrm{U}, \mathrm{M}$ with our $\mathrm{M}$ and $\mathrm{P}$, with $\mathrm{M}=\mathrm{P}$, and I with $\mathbf{T}$. As suggested, both views on theories seem to conceive them as maximal. However, one may also argue that they are purely dealing with an exclusion claim, viz. $(\mathbf{T}=) \mathrm{I} \subseteq \mathrm{M}(=\mathrm{P})$, in the structuralist case.
} 
Table 1 Truth- and falsity-content

\begin{tabular}{lll}
\hline Theory $<\mathrm{M}, \mathrm{P}>$ & M-side & $\mathrm{P}$-side \\
\hline M- resp. P-claim & $\mathrm{M} \subseteq \mathbf{T}$ & $\mathrm{T} \subseteq \mathrm{P}(\mathrm{cP} \subseteq \mathrm{cT})$ \\
Truth-content (TC) & $\mathrm{M} \cap \mathbf{T}$ & $\mathrm{cP} \cap \mathrm{cT}$ \\
Falsity-content (FC) & $\mathrm{M}-\mathbf{T}$ & $\mathrm{cP}-\mathrm{cT}$ \\
M- resp. P-content & $\mathrm{M}$ & $\mathrm{cP}$ \\
\hline
\end{tabular}

Fig. 4 Truth- and falsity-content. The indicated intersections constitute the relevant truth-content and the indicated difference sets the relevant falsity-content

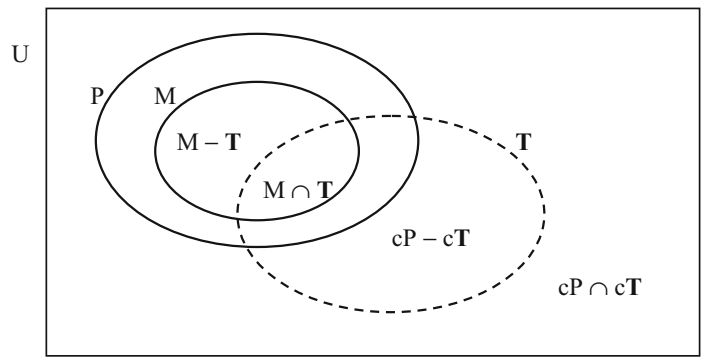

is concerned the sub-claim $\mathbf{M} \cap \mathbf{T} \subseteq \mathbf{T}$ is true and as far as $\mathbf{M}-\mathbf{T}$ is concerned the remaining sub-claim $\mathbf{M}-\mathbf{T} \subseteq \mathbf{T}$ is false. Hence we define $\mathrm{M} \cap \mathbf{T}$ as its truth-content and $\mathbf{M}-\mathbf{T}$ as its falsity-content. Note that their union equals $\mathrm{M}$, which can be seen as the (total) content of the M-side claim.

Similarly, regarding the $\mathrm{P}$-side claim $\mathbf{T} \subseteq \mathrm{P}$, that is, $\mathrm{cP} \subseteq \mathrm{cT}$, the sub-claim $\mathrm{cP} \cap \mathrm{c} \mathbf{T} \subseteq \mathrm{c} \mathbf{T}$ is true and the additional sub-claim $\mathrm{cP}-\mathrm{cT} \subseteq \mathrm{c} \mathbf{T}$ is false. Hence we define $\mathrm{cP} \cap \mathrm{cT}$ as its truth-content and $\mathrm{cP}-\mathrm{cT}$ as its falsity-content. Their union equals cP, in which Popper's idea of the empirical content can be recognized easily: the set of possibilities which are excluded by the postulates. Table 1 summarizes these definitions.

In combination with Table 1 and Fig. 4 makes graphically clear which subsets are involved.

\subsection{Closer to the truth (greater verisimilitude or truthlikeness)}

The notions of truth- and falsity-content just defined allow for a straightforward definition of "closer to the truth" in terms of greater truth-content and smaller falsity-content. Focusing first on pure M-side theories, for example, one can define:

$<\mathrm{M}^{*}, \mathrm{U}>$ is at least as close to the truth as the M-side theory $<\mathrm{M}, \mathrm{U}>$ iff TC-clause: the truth-content of $\langle\mathrm{M}, \mathrm{U}>$ is a subset of the truth-content of $<\mathrm{M}^{*}, \mathrm{U}>$ and

FC-clause: the falsity-content of $<\mathrm{M}^{*}, \mathrm{U}>$ is a subset of the falsity-content of $<\mathrm{M}, \mathrm{U}>$

A similar definition can of course be given for pure P-side theories. 
Table $2<\mathrm{M}^{*}, \mathrm{P}^{*}>$ is at least as close to the truth as $<\mathrm{M}, \mathrm{P}>$

\begin{tabular}{|c|c|c|}
\hline $\begin{array}{l}<\mathrm{M}^{*}, \mathrm{P}^{*}>\text { is at least as close } \\
\text { to the truth as }<\mathrm{M}, \mathrm{P}>\end{array}$ & M-side & P-side \\
\hline $\begin{array}{l}\text { TC-clauses: } \\
\text { TC is a subset of TC* }\end{array}$ & $\begin{array}{l}\mathbf{M} \cap \mathbf{T} \subseteq \mathrm{M}^{*} \cap \mathbf{T} \\
\quad \equiv \mathbf{T}-\mathbf{M}^{*} \subseteq \mathbf{T}-\mathbf{M}\end{array}$ & $\begin{array}{r}\mathrm{cP} \cap \mathrm{c} \mathbf{T} \subseteq \mathrm{cP}^{*} \cap \mathrm{cT} \\
\equiv \mathrm{P}^{*}-\mathbf{T} \subseteq \mathrm{P}-\mathbf{T} \\
\equiv \mathrm{P}^{*} \cup \mathbf{T} \subseteq \mathrm{P} \cup \mathbf{T}\end{array}$ \\
\hline $\begin{array}{l}\text { FC-clauses: } \\
\text { FC* is a subset of FC }\end{array}$ & $\mathrm{M}^{*}-\mathbf{T} \subseteq \mathrm{M}-\mathbf{T}$ & $\begin{array}{r}\mathrm{cP}^{*}-\mathrm{c} \mathbf{T} \subseteq \mathrm{cP}-\mathrm{c} \mathbf{T} \\
\equiv \mathbf{T}-\mathrm{P}^{*} \subseteq \mathbf{T}-\mathrm{P} \\
\quad \equiv \mathrm{P} \cap \mathbf{T} \subseteq \mathrm{P}^{*} \cap \mathbf{T}\end{array}$ \\
\hline Combined clauses & $\mathbf{M}^{*} \Delta \mathbf{T} \subseteq \mathrm{M} \Delta \mathbf{T}$ & $\mathrm{cP}^{*} \Delta \mathrm{cT} \subseteq \mathrm{cP} \Delta \mathrm{c} \mathbf{T}$ \\
\hline
\end{tabular}

Table 2 presents the combined definition of " $<\mathrm{M}^{*}, \mathrm{P}^{*}>$ is at least as close to the truth as $<\mathrm{M}, \mathrm{P}>$ ".

It is not difficult to check that the four single clauses are independent as long as the theories are non-maximal. Moreover, as indicated, on both sides the two single difference clauses can be combined into the corresponding combined symmetric-difference clauses. It is easy to check that for maximal theories $(\mathrm{M}=\mathrm{P})$ the two combined clauses are formally equivalent ${ }^{10,11}$.

Figure 5 illustrates " $<\mathrm{M}^{*}, \mathrm{P}^{*}>$ is at least as close to the truth as $<\mathrm{M}, \mathrm{P}>$ ". In combination with Table 2 the separated figures make graphically clear by shading which subsets have to be empty at the M-side (left) and at the P-side (right). Of course, the figures have to be conceived as combined, taking into account that $\mathrm{M} \subseteq \mathrm{P}$ and $\mathrm{M}^{*} \subseteq \mathrm{P}^{*}$. Hence, there will appear single and double shaded subareas.

Although the two figures look formally similar, it is important to note that corresponding shaded areas are empty due to 'opposite' clauses. E.g. in the left figure the shaded area on the left is empty due to the TC-clause for the M-side, viz. $\mathbf{M} \cap \mathbf{T} \subseteq \mathbf{M}^{*} \cap \mathbf{T}$, whereas in the right figure it is empty due to the FC-clause for the P-side, viz. $\mathrm{cP}^{*}-\mathrm{c} \mathbf{T} \subseteq \mathrm{cP}-\mathrm{cT}$, or, equivalently $\mathbf{T}-\mathrm{P}^{*} \subseteq \mathbf{T}-\mathrm{P}$.

It is also not difficult to check that the P-side can be summarized by 'having at least as many true consequences (for $\mathrm{P}^{*} \cup \mathbf{T}$ is included in $\mathrm{P} \cup \mathbf{T}^{12}$ ) and correctly allowing

10 The symmetric difference $\mathrm{A} \Delta \mathrm{B}$ is standardly defined as $(\mathrm{A}-\mathrm{B}) \cup(\mathrm{B}-\mathrm{A})$.

11 This leads to the original 'symmetric difference'- definition (Miller 1978; Kuipers 1982), assuming the strong claim of maximal theories: $\mathrm{M}=\mathrm{P}=\mathbf{T}$. By the way, Miller's definition was focused on 'the actual truth', i.e. one particular possibility. However, both share the characteristic that they are intuitively similar to Popper's original but faulty definition in terms of more true and less false consequences. The symmetric difference definition for maximal theories may be summarized as: (1) more correctly and less incorrectly included possibilities (as nomic) or, equivalently, (2) more correctly and less incorrectly excluded possibilities (from being nomic). In the generalized theory, applied to non-maximal theories, (1) characterizes 'closer to' on the M-side, and (2) on the P-side.

12 A true consequence of $<\mathrm{M}, \mathrm{P}>$ at the $\mathrm{P}$-side can be represented as a superset of both $\mathrm{P}$ and $\mathbf{T}$, i.e. a set having both $\mathrm{P}$ and $\mathbf{T}$ as subsets, for the following reason. Let $\mathrm{P}, \mathbf{T}$, and $\mathrm{C}$ (subset of $\mathrm{U}$ ) represent the models of the (complex) sentences $\mathrm{p}, \mathbf{t}$, and c, respectively, and let $\mathrm{P} \cup \mathbf{T} \subseteq \mathrm{C}$. Then $\mathrm{c}$ is a consequence of $\mathrm{p}$ and of $\mathbf{t}$, and hence a true consequence of $\mathrm{p}$, for $\mathrm{c}$ is true in all models of $\mathrm{P}$ and $\mathbf{T}$. The condition that $\mathrm{P}^{*} \cup \mathbf{T}$ is included in $\mathrm{P} \cup \mathbf{T}$ now guarantees that all supersets of $\mathrm{P} \cup \mathbf{T}$ are supersets of $\mathrm{P}^{*} \cup \mathbf{T}$, and hence 

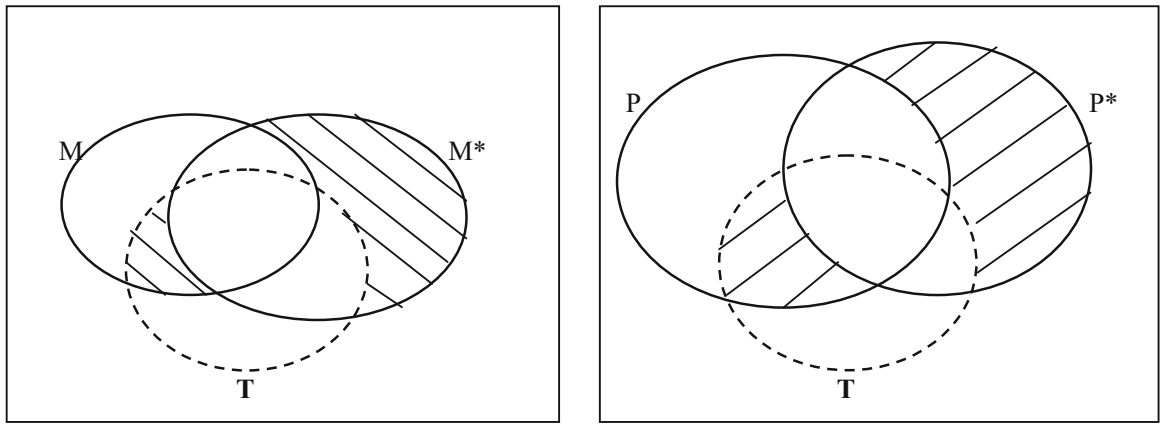

Fig. $5<\mathrm{M}^{*}, \mathrm{P}^{*}>$ is at least as close to the truth as $<\mathrm{M}, \mathrm{P}>$ : shaded areas are empty

at least as many possibilities ( $\mathrm{P}^{*} \cap \mathbf{T}$ includes $\mathrm{P} \cap \mathbf{T}$ ) and, conversely, the M-side by 'having at least as many correct models $\left(\mathrm{M}^{*} \cap \mathbf{T}\right.$ includes $\left.\mathrm{M} \cap \mathbf{T}\right)$ and allowing at least as many true consequences (for $\mathbf{M}^{*} \cup \mathbf{T}$ is included in $\mathbf{M} \cup \mathbf{T}$ )'.

Finally, it is plausible to define 'closer to the truth' if, in addition to 'at least as close to the truth', at least one of the four required single set-theoretic inclusions can be replaced by a proper inclusion and hence if at least one of the two required combined inclusions in terms of symmetric differences is proper.

\section{III Epistemological steps: define more successful}

\subsection{Empirical data}

So far, we have been engaged with the logical problem of verisimilitude, that is, explicating 'closer to the truth', assuming that we dispose of the truth in one way or another. Now we turn to the epistemological problem of specifying empirical conditions that support the conclusion, at least for the time being, that one theory is closer to the unknown truth than another. As we will see, empirical progress is such a condition.

In the nomic context the empirical data are asymmetric in the following sense (cf. Kuipers 2000, p. 157). We can establish by experiments nomic possibilities. In fact, every experiment realizes by definition a nomic possibility. However, it is evident that we cannot establish nomic impossibilities in such a direct way. But in the empirical sciences we use to 'induce' nomic impossibilities indirectly by inductive (empirical) generalizations that we accept, for the time being, on the basis of 'sufficient' experimentation, notably by trying to realize counterexamples. For example, the observation of a black raven is the realization of a nomic possibility, whereas concluding that nonblack ravens do not exist is an inductive generalization, viz. all ravens are black.

Footnote 12 continued

that all true consequences of $<\mathrm{M}, \mathrm{P}>$ (at the $\mathrm{P}$-side) are (true) consequences of $<\mathrm{M}^{*}, \mathrm{P}^{*}>$. For a detailed comparison between Popper's failing consequence-based approach and the 'model-based' approach, both for maximal theories, the reader is referred to Kuipers (2000, Chap. 8.1), where the latter is also translated in terms of consequences, leading to the identification of Popper's bad luck. 
Fig. 6 Data-theory $<\mathrm{R}, \mathrm{S}>$ depicted as a true theory

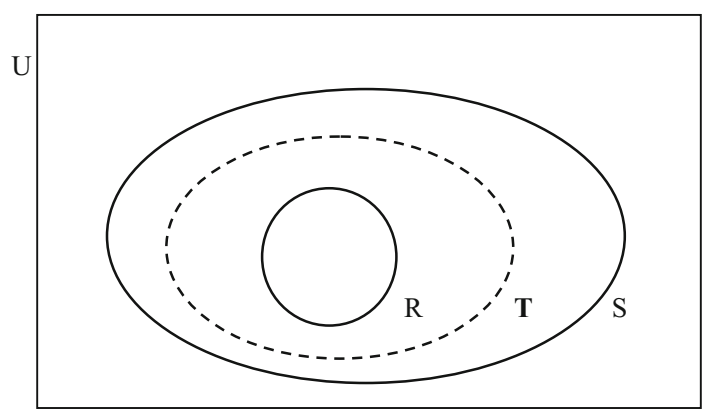

We indicate the (asymmetric) data at a certain moment by $<\mathrm{R}, \mathrm{S}>$, where $\mathrm{R}$ indicates the set of realized nomic possibilities (e.g. realized physical possibilities) and $\mathrm{S}$ indicates the strongest law induced on the basis of $\mathrm{R}$, more precisely, the set of conceptual possibilities that are not excluded by (the combination of) the accepted inductive generalizations. In this setup cS indicates the set of induced nomic impossibilities. Of course, we may always assume that $\mathrm{R} \subseteq \mathrm{S}$, for, if not, any element in R-S would represent a realized counterexample to $S$ and hence to at least one of its constituting inductive generalizations. Moreover, if the experiments are correctly described by $\mathrm{R}$ (relative to the vocabulary) and if, in addition, $\mathrm{S}$ is correctly induced, we may conclude that $\mathrm{R} \subseteq \mathbf{T} \subseteq \mathrm{S}$, whatever $\mathbf{T}$ is. Hence, we not only assume that $\langle\mathrm{R}, \mathrm{S}>$ is a theory, a 'data-theory', but by accepting it we even assume that it is a true theory, that is, we have accepted the claims $\mathrm{R} \subseteq \mathbf{T}$ and $\mathbf{T} \subseteq \mathrm{S}$ ( $\equiv \mathrm{cS} \subseteq \mathrm{cT}$ ). Of course, the correctness assumptions are very substantial, in particular that of correct inductive generalizations.

Figure 6 displays $<\mathrm{R}, \mathrm{S}>$ as a true theory.

\subsection{Accepted and rejected content}

To get plausible definitions of accepted and rejected content of a theory $<\mathrm{M}, \mathrm{P}>$ in the light of an accepted data-theory $<\mathrm{R}, \mathrm{S}>$ we have to confront the claims of the former, that is, $\mathrm{M} \subseteq \mathbf{T}$ and $\mathrm{cP} \subseteq \mathrm{cT}$, with the accepted claims of the latter, that is, with the claims $\mathrm{R} \subseteq \mathbf{T}$ and $\mathrm{cS} \subseteq \mathrm{c} \mathbf{T}$. Let us first look at the M-side. Of the theory claim $\mathrm{M} \subseteq \mathbf{T}$, recall, with content $\mathrm{M}$, we have, by accepting $\mathrm{R} \subseteq \mathbf{T}$, accepted the sub-claim $\mathrm{M} \cap \mathrm{R} \subseteq \mathbf{T}$, and hence the accepted M-content is $\mathrm{M} \cap \mathrm{R}$, which may be called the set of realized examples. ${ }^{13}$ However, by accepting $\mathrm{cS} \subseteq \mathrm{cT}$, we have also rejected the sub-claim $\mathrm{M} \cap \mathrm{cS} \subseteq \mathbf{T}$, and hence the rejected $M$-content is $\mathrm{M} \cap \mathrm{cS}(=\mathrm{M}-\mathrm{S})$, which may be called the set of induced counterexamples.

Similarly for the P-side. Of the theory claim $\mathrm{cP} \subseteq \mathrm{cT}$, recall, with content $\mathrm{cP}$, we have, by accepting $\mathrm{cS} \subseteq \mathrm{cT}$, accepted the sub-claim $\mathrm{cP} \cap \mathrm{cS} \subseteq \mathrm{cT}$, and hence the accepted $P$-content is $\mathrm{cP} \cap \mathrm{cS}$, which may be called the set of induced examples. However, by accepting $\mathrm{R} \subseteq \mathbf{T}$, we have also rejected the sub-claim $\mathrm{cP} \cap \mathrm{R} \subseteq \mathrm{cT}$,

13 This and the following '(counter-) example terminology' is a variation of similar terminology in Kuipers (2000, p. 158). 
Table 3 Accepted and rejected M- and P-content of theory $<\mathrm{M}, \mathrm{P}>$ in the light of accepted data-theory $<\mathrm{R}, \mathrm{S}>$

\begin{tabular}{|c|c|c|}
\hline $\begin{array}{l}<\mathrm{M}, \mathrm{P}>\text { in the light of } \\
<\mathrm{R}, \mathrm{S}>\end{array}$ & M-side & P-side \\
\hline M- resp. P-claim & $\mathbf{M} \subseteq \mathbf{T}$ & $\mathbf{T} \subseteq \mathrm{P}(\mathrm{cP} \subseteq \mathrm{c} \mathbf{T})$ \\
\hline Accepted claims & \multicolumn{2}{|c|}{$\mathbf{T} \subseteq \mathrm{S}(\mathrm{cS} \subseteq \mathrm{cT})$} \\
\hline $\begin{array}{l}\text { M-resp. P-content (of the } \\
\text { theory) }\end{array}$ & $\mathrm{M}$ & $\mathrm{cP}$ \\
\hline $\begin{array}{l}\text { Accepted (M- resp. P-) } \\
\text { content }\end{array}$ & $\begin{array}{l}\text { 1: } \mathrm{M} \cap \mathrm{R} \\
\quad \text { realized examples } \\
\text { (true positives) }\end{array}$ & $\begin{array}{l}\text { 4: } \mathrm{cP} \cap \mathrm{cS} \\
\text { induced examples } \\
\text { (true negatives) }\end{array}$ \\
\hline $\begin{array}{l}\text { Rejected (M- resp. P-) } \\
\text { content }\end{array}$ & $\begin{array}{l}\text { 2: } \mathrm{M}-\mathrm{S}(\mathrm{M} \cap \mathrm{cS}) \\
\quad \text { induced } \\
\text { counterexamples } \\
\quad \text { (false positives) }\end{array}$ & $\begin{array}{l}\text { 5: } \mathrm{cP} \cap \mathrm{R}(\mathrm{R}-\mathrm{P}) \\
\quad \text { realized } \\
\text { counterexamples } \\
\text { (false negatives) }\end{array}$ \\
\hline $\begin{array}{l}\text { Undecided (M-resp P-) } \\
\text { content }\end{array}$ & $3: \mathrm{M}-(\mathrm{R} \cup \mathrm{cS})$ & 6: $\mathrm{cP}-(\mathrm{R} \cup \mathrm{cS})$ \\
\hline
\end{tabular}

and hence the rejected $P$-content is $\mathrm{cP} \cap \mathrm{R}(=\mathrm{R}-\mathrm{P})$, which may be called the set of realized counterexamples.

Table 3 gives a full survey, adding the also plausible terminology of 'true and false positives (negatives)' ${ }^{14}$ the undecided content on both sides, ${ }^{15}$ and a numbering of the most relevant subsets.

Figure 7 illustrates all these concepts graphically, using the numbering of subsets in Table 3.

\subsection{At least as successful relative to $<R, S>$}

It is rather plausible to define now the idea that (the revised or new) theory $<\mathrm{M}^{*}, \mathrm{P}^{*}>$ is at least as successful as (the initial) theory $<\mathrm{M}, \mathrm{P}>$, relative to accepted data-theory $<\mathrm{R}, \mathrm{S}>$, by requiring, that all successes (realized and induced examples) of $<\mathrm{M}, \mathrm{P}>$ are successes of $<\mathrm{M}^{*}, \mathrm{P}^{*}>$ and all failures (induced and realized counterexamples) of $<\mathrm{M}^{*}, \mathrm{P}^{*}>$ are failures of $<\mathrm{M}, \mathrm{P}>$. Or, equivalently, at least as much accepted content (AC-clauses) and at most as much rejected content (RC-clauses) of the *theory on both sides, of course, not in terms of numbers but of subset conditions. The result is given in Table 4 .

\footnotetext{
14 True (false) positives are truly (falsely) claimed nomic possibilities, true (false) negatives are truly (falsely) claimed nomic impossibilities. This terminology assumes that we take the claim that an item in $\mathrm{U}$ is a nomic possibility as the claim to be 'a positive (result)' and that it is a nomic impossibility as the claim to be 'a negative (result)'. Now, for example, regarding the members of $\mathrm{M} \cap \mathrm{R}$, theory $<\mathrm{M}, \mathrm{P}>$ truly claims that they are positives, hence they may be called 'true positives'.

15 The undecided M-content is that part of the content of $M$ about which $<R, S>$ is silent, that is, the $\mathrm{M}$-content apart from its accepted and rejected parts: 'M-content - (accepted M-content $\cup$ rejected Mcontent $)=\mathrm{M}-[(\mathrm{M} \cap \mathrm{R}) \cup(\mathrm{M} \cap \mathrm{cS})]=\mathrm{M}-(\mathrm{R} \cup \mathrm{cS})$. Similarly for the undecided P-content.
} 


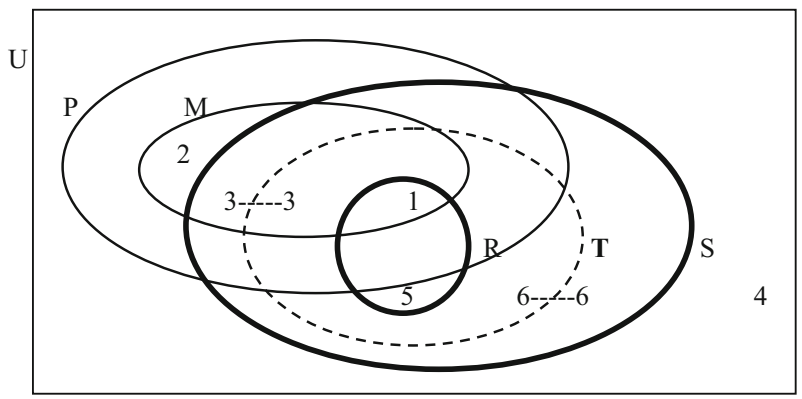

Fig. 7 Accepted and rejected $\mathrm{M}$ - and P-content of theory $<\mathrm{M}, \mathrm{P}>$ in the light of accepted data-theory $<\mathrm{R}, \mathrm{S}>$. See Table 3 for the legend. Note It may be interesting to note that P-S represents the wrongly not excluded nomic impossibilities, of which the subset M-S represents the wrongly included nomic impossibilities

Table $4<\mathrm{M}^{*}, \mathrm{P}^{*}>$ is at least as successful as $<\mathrm{M}, \mathrm{P}>$, relative to $<\mathrm{R}, \mathrm{S}>$

\begin{tabular}{|c|c|c|}
\hline $\begin{array}{l}<\mathrm{M}^{*}, \mathrm{P}^{*}>\text { is at least as } \\
\text { success-ful as }<\mathrm{M}, \mathrm{P}>\text {, } \\
\text { relative to }<\mathrm{R}, \mathrm{S}>\end{array}$ & M-side & P-side \\
\hline $\begin{array}{l}\text { AC-clauses: } \mathrm{AC} \text { is a } \\
\text { subset of } \mathrm{AC}^{*}\end{array}$ & $\begin{array}{l}\mathrm{M} \cap \mathrm{R} \subseteq \mathrm{M}^{*} \cap \mathrm{R} \\
\equiv \mathrm{R}-\mathrm{M}^{*} \subseteq \mathrm{R}-\mathrm{M} \\
\text { all realized examples of } \\
<\mathrm{M}, \mathrm{P}>\text { are examples } \\
\text { of }<\mathrm{M}^{*}, \mathrm{P}^{*}>\end{array}$ & $\begin{array}{l}\mathrm{cP} \cap \mathrm{cS} \subseteq \mathrm{cP}^{*} \cap \mathrm{cS} \\
\equiv \mathrm{P}^{*}-\mathrm{S} \subseteq \mathrm{P}-\mathrm{S} \\
\text { all induced examples of } \\
<\mathrm{M}, \mathrm{P}>\text { are examples } \\
\text { of }<\mathrm{M}^{*}, \mathrm{P}^{*}>^{\mathrm{a}}\end{array}$ \\
\hline $\begin{array}{l}\text { RC-clauses: } \mathrm{RC}^{*} \text { is a } \\
\text { subset of } \mathrm{RC}\end{array}$ & $\begin{array}{l}\mathrm{M}^{*}-\mathrm{S} \subseteq \mathrm{M}-\mathrm{S} \\
\text { all induced } \\
\text { counterexamples of } \\
<\mathrm{M}^{*}, \mathrm{P}^{*}>\text { are } \\
\text { counterexamples of } \\
<\mathrm{M}, \mathrm{P}>\end{array}$ & $\begin{array}{l}\mathrm{cP}^{*}-\mathrm{cR} \subseteq \mathrm{cP}-\mathrm{cR} \\
\equiv \mathrm{R}-\mathrm{P}^{*} \subseteq \mathrm{R}-\mathrm{P} \\
\text { all realized } \\
\text { counterexamples of } \\
<\mathrm{M}^{*}, \mathrm{P}^{*}>\text { are } \\
\text { counterexamples of } \\
<\mathrm{M}, \mathrm{P}>\end{array}$ \\
\hline
\end{tabular}

\footnotetext{
a Since the clause is also equivalent to $\mathrm{S} \cup \mathrm{P}^{*} \subseteq \mathrm{S} \cup \mathrm{P}$ it can also be paraphrased by "all induced laws entailed by $<\mathrm{M}, \mathrm{P}>$ are entailed by $<\mathrm{M}^{*}, \mathrm{P}^{*}>$, for any superset of $\mathrm{S} \cup \mathrm{P}$ is a superset of $\mathrm{S} \cup \mathrm{P}^{*}$. See Note 12
}

Of course, 'more successful' is defined by requiring, in addition, that at least one of the four single clauses is a proper inclusion.

\section{Remaining steps: from verisimilitude to success, and vice versa}

At this point we just repeat the remaining steps in setting up a theory of nomic truth approximation and briefly give some particular features of the specific case.

IV Theoretical step: from verisimilitude to success

- Assuming the truth of the (accepted) evidence, prove the strongest 'success theorem'. Ideally this theorem amounts to: 'closer to the truth' unconditionally entails 'at least as successful' and in the long run even 'more successful'.

In the present case it is not difficult to check that 'ideally' applies, i.e. if $<\mathrm{M}^{*}, \mathrm{P}^{*}>$ is closer to the truth than $<\mathrm{M}, \mathrm{P}>$ and if the data-theory $<\mathrm{R}, \mathrm{S}>$ is 
true, this entails that $<\mathrm{M}^{*}, \mathrm{P}^{*}>$ is at least as successful as $<\mathrm{M}, \mathrm{P}>$ relative to $<\mathrm{R}, \mathrm{S}>$, and, under some probabilistic test assumptions, ${ }^{16}$ that it will become more successful in the long run.

V Methodological steps: from success to verisimilitude

- Assuming that a new theory is at a certain moment more successful than an old one, propose and test the empirical progress hypothesis: the new theory (is and) remains more successful than the old one.

Note that the two claims of a theory lead to different kinds of predictions:

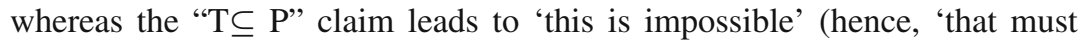
happen') predictions, the " $\mathrm{M} \subseteq \mathrm{T}$ "-claim leads to 'this is possible / may happen'-predictions. With plausible consequences for differential predictions between $<\mathrm{M}, \mathrm{P}>$ and $<\mathrm{M}^{*}, \mathrm{P}^{*}>$.

- Assuming that after 'sufficient confirmation' the empirical progress (EP-)hypothesis is accepted (for the time being), which is an inductive conclusion, argue on the basis of the success theorem that the best explanation for this case of empirical progress is the truth approximation (TA-) hypothesis that the new theory is closer to the truth than the old one, i.e. that this is a case of truth approximation.

The reverse ('from success to verisimilitude') consequences of the success theorem, i.e. the consequences of that theorem in view of an accepted EPhypothesis (and the data-theory on which it is based), are such that this situation not only suggests the TA-hypothesis, they also justify it to a substantial extent: If $<\mathrm{M}^{*}, \mathrm{P}^{*}>$ is, in view of $<\mathrm{R}, \mathrm{S}>$, accepted as empirically progressive relative to $<\mathrm{M}, \mathrm{P}>$, then

1. the success theorem not only makes it perfectly possible that $<\mathrm{M}^{*}, \mathrm{P}^{*}>$ is closer to the truth than $<\mathrm{M}, \mathrm{P}>$, due to it the TA-hypothesis would even explain the greater success,

2. it is impossible that $<\mathrm{M}^{*}, \mathrm{P}^{*}>$ is further from the truth than $<\mathrm{M}, \mathrm{P}>$ (and hence $<\mathrm{M}, \mathrm{P}>$ closer to the truth than $<\mathrm{M}^{*}, \mathrm{P}^{*}>$ ), for otherwise, so shows the success theorem, $<\mathrm{M}^{*}, \mathrm{P}^{*}>$ could not be more successful,

3. it is also possible that $<\mathrm{M}^{*}, \mathrm{P}^{*}>$ is neither closer to nor further from the truth than $<\mathrm{M}, \mathrm{P}>$, in which case, however, another specific explanation has to be given for the fact that $<\mathrm{M}^{*}, \mathrm{P}^{*}>$ has so far proven to be more successful, e.g. due to a biased choice of experiments.

Note that the TA-hypothesis provides a typical default explanation of EP, that is, an adequate explanation unless there turns out to be reason for another diagnosis. The third reverse consequence provides the room for 'unless' conditions

\footnotetext{
16 E.g. suppose $\mathrm{U}$ is finite and let $<\mathrm{M}^{*}, \mathrm{P}^{*}>$ be closer to the truth than $<\mathrm{M}, \mathrm{P}>$ (partly) due to the 'strong' TC-clause (see Table 2) that $\mathbf{M} \cap \mathbf{T}$ is a proper subset of $\mathbf{M}^{*} \cap \mathbf{T}$. If $\mathbf{R}$ results from random sampling in $\mathrm{T}$, sooner or later one of the extra items in $\mathbf{M}^{*} \cap \mathbf{T}$ (i.e. items in $\left.\left(\mathbf{M}^{*} \cap \mathbf{T}\right)-\mathbf{M}\right)$ will show up, proving 'more successful'. Similarly, for the possible strong TC-clause on the P-side and the two possible strong FC-clauses.
} 
and hence for future 'divided success', i.e. the old theory may get extra successes relative to the new one. For example, the experiments so far may turn out to have been biased in favor of the new theory, and hence new experiments breaking this bias may turn out to be in favor of the old one.

- Abductively conclude (for the time being) that the new theory is closer to the truth than the old one, i.e. that truth approximation has been achieved.

This final step will now be no surprise. In fact, it is a special case of a sophisticated form of 'inference to the best explanation' or 'inference to the best theory', viz. not as a, or even the, true theory, but as the theory which is the closest to the truth of the available theories. Of course, 'inference to the best theory' should be read as: inference to the best theory of all available theories beyond the data-theory $<\mathrm{R}, \mathrm{S}>{ }^{17}$

\section{Perspectives}

To be sure, the above analysis is based on the simplest assumptions about the further nature of theories and their claims, for which reason I call it the basic version of the generalized theory. In this section I will briefly indicate four perspectives for concretization, as far as relevant, in line with those of Kuipers (2000). I will conclude with indicating an alternative interpretation and a connection with belief revision.

\subsection{Refinement, capturing idealization and concretization}

There are two plausible qualitative concretizations of the basic version of the two-sided approach, refinement and stratification (cf. Kuipers 2000, Chaps. 9-10, respectively). Refinement makes it possible to account for the fact that one counterexample may be less severe than another, e.g. in the sense that it is less idealized than the other. In Kuipers (2000, Chap. 10) I have presented such a refined approach to empirical progress and (nomic) truth approximation based on an underlying ternary similarity relation between possibilities, that is, structures, and hence called a structurelikeness relation. The relation "one structure is more similar to a third than another structure" may in particular take the form "one structure is less idealized, relative to a still more realistic structure, than another". In this way it was possible to explicate the idea of 'truth approximation by idealization and concretization'. ${ }^{18}$ Of course, the point of departure was then the strong claim, combining the inclusion and the exclusion method in an extreme complementary way.

\footnotetext{
17 The latter has to be excluded, for it is not only assumed to be true, it would provide a circular explanation of being the best theory. Moreover, opting for it would also amount to the conclusion that after all there was nothing of interest in the original theory.

18 Idealization is an extremely important aspect of scientific theorizing, as for example Nowak (1980), Cartwright (1983) and, more recently, Shaffer (2012) have argued and documented. My view on idealization in relation to truth approximation is particularly guided by Leszek Nowak's emphasis on successive concretization, which can be explicated as aiming at truth approximation
} 
From the present two-sided perspective, the refined definitions of 'closer to the truth' and 'more successful' seem to be primarily concretizations of the clauses corresponding to the representation function of inclusion theories. In the refined inclusion method new theories revise old theories by changing the models of the theories to some extent, e.g. by taking new factors into account that have been neglected before. The new models are claimed to be more similar to 'the true ones' than those before. Hence, although the exclusion method may appeal to Popperian intuitions, revising theories in the suggested refined way seems to reflect the representation part of scientific common sense. Therefore, it is plausible to think that an adequate modeling of scientific common sense has to take into account both intuitions. This is perfectly possible from the present perspective: two-sided theories for which the basic clauses are used for the exclusion subtheories, whereas the refined clauses are used for the inclusion subtheories. In terms of Zwart (2001), this is a way of combining the (Popperian) 'content-approach', with the 'similarity approach' to truth approximation, à la Niiniluoto (1987) and Oddie (1986). One may even speculate that Lakatosian research program thinking can be represented by such two-sided theories: progress is achieved by revising the corresponding inclusion theory more in particular by revising, e.g. concretizing, auxiliary hypotheses, however within the boundaries of the corresponding exclusion theory, forming the hard core. The suggested asymmetric way of dealing with refinement may seem plausible, in the practice of science refinement occurs on both sides. E.g. Einstein's postulates refine, relative to Newton's postulates, which possibilities are excluded from being realizable.

Since it is perfectly possible to translate the refinement of the model-side of theories to their postulates, by taking suitable complements, leading to the refinement of the postulate side, we prefer to conceive the refinement of truthlikeness of two-sided theories primarily in a symmetric way (Kuipers, manuscript). In this symmetric version, 'closer to the truth' is, roughly, defined by requiring on both sides a larger truth-content and a less problematic falsity-content, the latter in the sense that the falsity-content of the one theory is, in terms of the structurelikeness relation, more similar to (part of) the truth than the falsity-content of the other. From this symmetric version it is easy to derive the above suggested asymmetric version by 'idealizing' the exclusion side in order to get its basic version back. This can be done by assuming a 'trivial' similarity relation. Finally, it turns out that 'more successful' can best be refined in a somewhat weaker, but more plausible way than before, with the attractive consequence that the Success Theorem remains unconditionally valid: truth approximation in the refined sense entails at least as successfulness in the refined sense.

\subsection{Quantification}

It is important to note that 'closer to the truth' and 'more successful' in all forms so far dealt with are partial order relations. Hence, even in the basic version theories will frequently not be comparable in either direction. There are at least two plausible way-outs. From a methodological point of view it seems important to have a strategy to deal with cases of 'divided success', that is, when the one theory is more successful in some respects and the other in other respects. The qualitative ideal suggests to try 
to apply in this situation a kind of 'principle of dialectics', that is: try to improve both theories in one stroke. In other words, try to design a new theory, a synthesis, that is and remains more successful than both, that is, try to achieve genuine empirical progress, and hence, presumably, truth approximation with respect to both theories.

Another way to deal with the non-comparability problem is to design a quantitative concretization, in the present context, to begin with of the basic version. In a finite context it is even plausible to just count number of elements, leading to the quantitative symmetric difference definition of 'closer to the truth', e.g. referring to the P-side $\left|\Delta\left(\mathrm{P}^{*}, \mathbf{T}\right)\right| \leq|\Delta(\mathrm{P}, \mathbf{T})|$. However, as soon as one wants to differentiate between the weight of 'successes' and 'failures' or if U is infinite, ad hoc elements, e.g. weighing factors and other parameters, are unavoidable, witness Niiniluoto's otherwise impressive approach (Niiniluoto 1987). In Kuipers (manuscript) I nevertheless present a general quantitative (two-sided basic) approach, viz. a so-called measure-theoretical one. It is largely in the spirit of Kuipers (2000, Chap. 12). It leads almost always to an ordering of two theories, however, with a non-deductive 'success theorem', in terms of expectation values, about the relation between truth approximation and the corresponding quantitative notion of empirical progress. But after sufficient confirmation of the corresponding empirical progress hypothesis, the theorem will support the abductive 'closer to the truth' conclusion substantially.

\subsection{Stratification}

The second important qualitative concretization of the basic version of the two-sided approach deals with stratification in terms of an observational and a theoretical level. It is more or less crucial for the realism/instrumentalism debate. In Kuipers (2014b) I have already presented stratification for exclusion theories, in line with Kuipers (2000, Chap. 9). It leads to some substantial weakening of the connection between empirical progress and truth approximation, but the connection remains remarkable. In Kuipers (manuscript) stratification for two-sided theories is elaborated. The crucial question for both sides is to what extent 'closer to the truth' on the theoretical level is projected on the observational level. Though formally similar, the possible exceptions have different methodological impact for the two sides, due to the asymmetric nature of evidence in the nomic context. Of course, stratification is also possible for the refined and quantified versions of 'closer to the truth', with specific limits to its projection on the observational level.

\subsection{Inconsistent theories}

Inconsistent two-sided theories, that is, theories where $\mathrm{M}$ is not a subset of $\mathrm{P}$ and hence some models are excluded by the postulates, may be based on good reasons for the models as well as for the postulates. Such theories may well be very useful for truth approximation. To begin with, starting with inconsistent theory $<\mathrm{M}, \mathrm{P}>$ one may be heading for a consistent theory $<\mathrm{M}^{*}, \mathrm{P}^{*}>$ such that the latter is 'side-wise' closer to the truth than the former. And even an inconsistent two-sided theory may be side-wise closer to the truth than another one, and hence be a step in the direction of the truth. 
This is formally perfectly possible. However, the question is to what extent $<\mathrm{M}, \mathrm{P}>$ can still be meaningfully considered to be one theory, though inconsistent. How can $\mathrm{M}$ and $\mathrm{P}$ still be substantially related, i.e., share more than the vocabulary, when the models do not satisfy (all of) the postulates? Of course, one option is that the models may satisfy some approximate version of the postulates or only the most fundamental postulates. This is clearly something which needs to be investigated further.

I like to conclude with two other perspectives.

\subsection{A monadic existential and a monadic nomic interpretation}

The formal story with the above 'nomic' interpretation can easily be given a monadic (existential) interpretation, ${ }^{19}$ in which the members of $U$ represent Q-predicates. ${ }^{20} \mathrm{~A}$ theory $<\mathrm{M}, \mathrm{P}>$ now says that the members of $\mathrm{M}$ are instantiated and the members of $\mathrm{cP}$ are not, i.e. a theory corresponds to a 'partial or complete constituent' in the standard logical sense. However, not only a monadic existential interpretation is possible, but also a monadic nomic interpretation: $<\mathrm{M}, \mathrm{P}>$ is then assumed to be claiming that it is nomically possible to instantiate the Q-predicates in M but not those in cP. The periodic table of elements can be seen as an example of the first interpretation, but even better of the latter.

\subsection{Connection with belief revision}

The main message of Kuipers (2014a) ${ }^{21}$ is that Sven Owe Hansson's adaptation of AGM-rules for belief base revision (BRR) provides adequate means to connect belief revision with a very general form of basic truth approximation. ${ }^{22}$ From the perspective of the present paper it is now not difficult to derive from that paper how revision of two-sided theory $<\mathrm{M}, \mathrm{P}>$ in the light of data theory $<\mathrm{R}, \mathrm{S}>$ can be reconstructed as a combination of expansion and retraction. Assuming both theories to be consistent, it is easy to check that by contraction, i.e. weakening, of the claims of $<\mathrm{M}, \mathrm{P}>$ as far as in conflict with those of $<\mathrm{R}, \mathrm{S}>$, we get the two-sided revised theory $<\mathrm{M} \cap \mathrm{S}, \mathrm{P} \cup \mathrm{R}>$. By successive expansion, i.e. strengthening, of the claims of this intermediate theory by the extra claims of $<\mathrm{R}, \mathrm{S}>$ relative to those of $<\mathrm{M}, \mathrm{P}>$ we get $<(\mathrm{M} \cap \mathrm{S}) \cup \mathrm{R},(\mathrm{P} \cup \mathrm{R}) \cap \mathrm{S}>$. It is also not difficult to check that the final theory is not only more successful than the original and the intermediate one (it is even maximally successful), but also that it is even closer to the truth than both, assuming of course that the data theory is true: $\mathrm{R} \subseteq \mathbf{T} \subseteq \mathrm{S}$. Further investigation is needed to connect the refined version of two-sided

19 In Kuipers (2014a), see below, I have already mentioned this interpretation.

20 Carnap's term for mutually exclusive and jointly exhaustive predicates, arising from a set of primitive predicates by all possible combinations of negated and unnegated ones.

21 A very general paper also inspired by Cevolani et al. (2011), but before realizing the liberating consequences for nomic truth approximation, let alone its room for truth approximation by two-sided theories connecting the model and the postulate views on theories.

22 And less complicated than the connection with belief set revision in AGM-style, as elaborated in Kuipers (2011). 
nomic truth approximation, suggested above, and a correspondingly refined version of belief base revision.

Acknowledgments I like to thank David Atkinson, Erik Krabbe, and Jan-Willem Romeijn for their helpful remarks at a first presentation in Groningen (PCCP), Fred Muller as commentator and the rest of the audience in Rotterdam (EIPE), the audience in Cambridge (CamPoS), in Bayreuth (Research Forum) and in Paris (CNRS) and Roberto Festa and, above all, Gustavo Cevolani for their constructive comments. Finally, I would like to thank three anonymous referees for their comments and suggestions.

Open Access This article is distributed under the terms of the Creative Commons Attribution 4.0 International License (http://creativecommons.org/licenses/by/4.0/), which permits unrestricted use, distribution, and reproduction in any medium, provided you give appropriate credit to the original author(s) and the source, provide a link to the Creative Commons license, and indicate if changes were made.

\section{References}

Cartwright, N. (1983). How the laws of physics lie. Oxford: Clarendon Press.

Cevolani, G., Crupi, V., \& Festa, R. (2011). Verisimilitude and belief change for conjunctive theories. Erkenntnis, 75(2), 183-222.

Cevolani, G., Festa, R., \& Kuipers, T. (2013). Verisimilitude and belief change for nomic conjunctive theories. Synthese, 190(16), 3307-3324.

Deutsch, D. (2013). Constructor theory. Synthese, 190(18), 4331-4359. doi:10.1007/s11229-013-0279-z.

Kuipers, T. (1982). Approaching descriptive and theoretical truth. Erkenntnis, 18(3), 343-378.

Kuipers, T. (Ed.). (1987). What is closer-to-the-truth? A parade of approaches to truthlikeness. Amsterdam: Rodopi.

Kuipers, T. (2000). From instrumentalism to constructive realism. Dordrecht: Kluwer AP.

Kuipers, T. (2011). Basic and refined nomic truth approximation by evidence-guided belief revision in AGM-terms. Erkenntnis, 75(2), 223-236.

Kuipers, T. (2014a). Dovetailing belief base revision with (basic) truth approximation. In E. Weber, D. Wouters, \& J. Meheus (Eds.), Logic, reasoning, and rationality (pp. 77-93). Dordrecht: Springer.

Kuipers, T. (2014b). Empirical progress and truth approximation revisited. Studies in History and Philosophy of Science, 46, 64-72.

Kuipers, T. (manuscript) Truth approximation revisited.

Miller, D. (1974). Popper's qualitative theory of verisimilitude. The British Journal for the Philosophy of Science, 25(2), 166-177.

Miller, D. (1978). On distance from the truth as a true distance. In J. Hintikka, et al. (Eds.), Essays on mathematical and philosophical logic (pp. 415-435). Dordrecht: Reidel.

Niiniluoto, I. (1987). Truthlikeness. Dordrecht: Reidel.

Niiniluoto, I. (1998). Verisimilitude: The third period. The British Journal for the Philosophy of Science, 49(1), 1-29.

Nowak, L. (1980). The structure of idealization. Dordrecht: Reidel.

Oddie, G. (1986). Likeness to truth. Dordrecht: Reidel.

Oddie, G. (2014). Truthlikeness. In E. N. Zalta (Ed.), The Stanford encyclopedia of philosophy (January, 2014 ed.). http://plato.stanford.edu/entries/truthlikeness.

Popper, K. R. (1963). Conjectures and refutations: The growth of scientific knowledge (3rd ed.). London: Routledge and Kegan Paul.

Shaffer, M. (2012). Counterfactuals and scientific realism. London: Palgrave Macmillan.

Tichý, P. (1974). On popper's definitions of verisimilitude. The British Journal for the Philosophy of Science, 25(2), 155-160.

Zwart, S. (2001). Refined verisimilitude. Dordrecht: Kluwer AP. 